\title{
Mola hidatiforme e doença trofoblástica gestacional
}

\author{
Hydatidiform mole and gestational trophoblastic disease
}

Palavras-chave

Mola hidatiforme/diagnóstico Mola hidatiforme/invasiva/diagnóstico Gonadotropina coriônica/ administração \& dosagem Gonadotropina coriônica humana subunidade beta/ administração \& dosagem

Keywords

Hydatidiform mole/diagnosis Hydatidiform mole, invasive/diagnosis Chorionic gonadotropin/ administration \& dosage Chorionic gonadotropin, beta subunit, human/administration \& dosage

Correspondêncio:

Jurandyr Moreira de Andrade Departamento de Ginecologia e Obstetrícia da Faculdade de Medicin de Ribeirão Preto da Universidade de São Paulo Avenida Bandeirantes, 3.900 CEP 14049-900 - Ribeirão Preto/SP Fone: (16) 3602-2803 E-mail: andrade@fmrp.usp.br

Recebido 23/12/08

\section{Resumo}

A mola hidatiforme é uma complicação relativamente infrequente da gravidez, mas com potencial para evolução para formas que necessitam de tratamento sistêmico e podem ser ameaçadoras da vida. Sob a denominação de mola hidatiforme há duas entidades histopatológicas e clínicas: a mola parcial e a completa. As diferenças entre estas duas formas são importantes, devido ao risco de evolução para formas persistentes, ou seja, mais alto para as completas. $\bigcirc$ diagnóstico da mola hidatiforme, seu tratamento e seguimento após o tratamento inicial sofreram alterações importantes nos últimos anos. $\bigcirc$ número de pacientes assintomáticas tem aumentado devido ao emprego de ultrassonografia no início da gravidez. Para a resolução da mola hidatiforme é necessário evitar o emprego de medicamentos que induzam contrações uterinas e usar a vácuo-aspiração. Deve ser prescrito o método contraceptivo hormonal logo após o esvaziamento da mola. $\bigcirc$ seguimento é baseado nas dosagens seriadas semanais de gonadotrofinas coriônicas. É importante que o método empregado detecte todas as formas das gonadotrofinas coriônicas (molécula intacta, hiperglicosilada, subunidade $\beta$ livre e fragmento central da subunidade $\beta$ ).

\section{Abstract}

The hydatiform mole is a relatively rare pregnancy complication, but with potential to evolve to forms which need systemic treatment and can be a threat to life. There are two histopathological and clinical entities under the name of hydatiform mole: the partial and the complete mole. The differences between the two forms are important due to risk of evolution to persistent forms, which is higher for the complete moles. The diagnosis, treatment and follow-up of hydatiform mole have been under important changes in the last years. The number of asymptomatic patients has increased, due to the use of ultrasonography at the onset of pregnancy. The use of medication that induces uterine contractions must be avoided, and vacuum aspiration should be used. Soon after emptying the mole, a hormonal contraceptive method should be prescribed. Follow-up should be based on weekly serial dosages of chorionic gonadotropin. It is important that the method employed detects all the forms of chorionic gonadotropins (intact molecule, with hyper glycol, free $\beta$ subunit, and central fragment $\beta$ subunit).
' Professor titular do Departamento de Ginecologia e Obstetrícia da Faculdade de Medicina de Ribeirão Preto da Universidade de São Paulo - USP - Ribeirão Preto (SP), Brasil. 


\section{Métodos para obtenção do texto}

Foi efetuada uma pesquisa não-sistemática das bases de dados Lilacs (166 trabalhos listados), SciELO e Pubmed empregando os termos mola hidatiforme $(\mathrm{MH})$ e doença trofoblástica. Para a revisão, foram selecionados os trabalhos completos dando preferência para os mais recentes sobre cada tópico. Foram incluídos apenas os trabalhos cujo texto integral fosse citado nas bases de dados mencionadas, sendo empregados os acessos permitidos pelo Portal Coordenação de Aperfeiçoamento de Pessoal de Nível Superior (CAPES) e no Sistema de Bibliotecas e Informação da Universidade de São Paulo (SIBI-USP). Não foram consultados os relatos de casos, trabalhos apresentados apenas como resumos, resumos apresentados em congressos e monografias. Para os pontos em que a opinião de especialistas fosse a única opção, foram consultados protocolos (guidelines) e revisões sobre tópicos específicos desde que a versão integral fosse de livre acesso. Nesta parte inicial da revisão serão abordados aspectos gerais da doença trofoblástica, diagnóstico da mola hidatiforme, tratamento inicial e seguimento pós-tratamento.

\section{Definição, epidemiologia, patologia e} classificação da doença trofoblástica

A MH é uma complicação da gravidez com potencial para evolução para doença com comportamento maligno e que ocorre, no Ocidente, numa proporção de um caso para 1.000 a 2.000 gestações $^{1}$, sendo a doença trofoblástica gestacional (DTG) mais frequente. Apenas um estudo brasileiro sobre o tema indica, com base em atendimento hospitalar de um único centro, a proporção de uma $\mathrm{MH}$ para 215 gestações $^{2}$.

A MH está incluída num grupo mais amplo conhecido genericamente como DTGs e que compreende, além da $\mathrm{MH}$, as formas com comportamento maligno como a neoplasia trofoblástica gestacional, o coriocarcinoma e o tumor trofoblástico de leito placentário, entre outras menos frequentes. São reconhecidos dois tipos de MH: completa (MHC) e parcial ou incompleta (MHP). Entre elas, há diferenças quanto aos aspectos morfológicos (macroscópi$\cos$ ), histopatologia e cariótipo ${ }^{3}$. Quanto à histopatologia, as MHC não têm elementos fetais, mostram proliferação generalizada e mais pronunciada do trofoblasto e maior frequência de atipias. Apesar da existência de critérios teóricos bem estabelecidos, há grande variação intra e interobservadores ${ }^{4}$. Além de elementos que dificultam o diagnóstico, como a ocorrência de gravidez gemelar com uma $\mathrm{MHC}^{5}$, pode ocorrer superposição de diagnósticos histológicos e as dificuldades são mais frequentes na distinção entre degeneração hidrópica (condição não relacionada à doença trofoblástica) e MHP, e entre MHP e $\mathrm{MHC}^{6}$.
Quanto ao cariótipo, a MHC é o resultado da fecundação de um óvulo sem núcleo ativo, o que significa que todos os genes na MHC são de origem paterna (dissomia uniparental). Entre as MHC, 90\% têm cariótipo 46, XX e os $10 \%$ restantes, $46, \mathrm{XY}$. Estas anomalias cromossômicas causam a perda precoce do embrião e proliferação excessiva do tecido trofoblástico. A MHP é associada ou causada por triploidia $(69 \mathrm{XXY})$ e mais raramente tetraploidia (92XXXY), com o conjunto de cromossomos extrahaplóide de origem paterna (diandria). Estas anomalias ocorrem quando um óvulo normal é fecundado por dois espermatozoides ou um espermatozoide diplóide ${ }^{7}$.

Do ponto de vista clínico, o volume uterino aumentado e complicações como hiperemese, pré-eclâmpsia e cistos tecaluteínicos são mais frequentes entre as portadoras de MHC. As pacientes com MHP geralmente apresentam sintomas consistentes com abortamento incompleto ou retido e por isto quase sempre o diagnóstico de MHP é obtido após avaliação histológica de material de curetagem. As diferenças são também notáveis quanto aos níveis de gonadotrofinas e, principalmente, para a taxa de evolução para doença persistente, que é de 18 a $28 \%$ contra 1 a $4 \%$ entre as $\mathrm{MHP}^{3}$.

A incidência de DTG e, especialmente de $\mathrm{MH}$, na população é baixa, mas são descritas várias famílias portadoras de $\mathrm{MH}$. Do ponto de vista individual, embora quase todas as portadoras de $\mathrm{MH}$ tenham futuro reprodutivo normal ${ }^{8}$, o risco aumenta após a primeira gestação molar, em até cinco vezes. Outras investigações apontam um risco ainda mais elevado ${ }^{3}$. Nesta investigação, a chance de repetição da $\mathrm{MH}$ foi de $1 \%$ e, comparada com a proporção estimada de um caso para 1.000 gestações, haveria aumento de dez vezes. Por este motivo, é justificável a avaliação ultrassonográfica precoce nas gestações subsequentes à $\mathrm{MH}$ para confirmar a presença e desenvolvimento normal do embrião.

\section{Forma de apresentação clínica da mola hidatiforme}

Um dos aspectos importantes da doença trofoblástica, pelo menos quanto a sua forma mais frequente (MHC), é a mudança da forma de apresentação nas últimas décadas ${ }^{9-11}$. Assim, quadros clínicos com molas de grande volume, eliminação de vesículas e anemia são cada vez menos frequentes $^{9}$. Situações de emergência tornaram-se mais raras, mas continuam a exigir atenção. Entre elas são ainda detectadas a insuficiência respiratória após o esvaziamento da mola, pré-eclâmpsia, hipertireoidismo e crise tireotóxica ${ }^{3}$.

Foram observadas outras mudanças na epidemiologia e forma de apresentação. Assim é relatada diminuição da média de idade das pacientes com MHC, além de menores volumes uterinos ${ }^{11}$, podendo esta última ser atribuída ao diagnóstico precoce. 
Em boa parte dos casos, portanto, o diagnóstico é efetuado em mulheres sem sintomas relacionados à mola, pelo menos nos países desenvolvidos ${ }^{11}$, sendo de se esperar maior porcentagem de casos sintomáticos nos países em desenvolvimento. Todas estas alterações no quadro clínico devem ser basicamente ao início precoce do pré-natal e, mais ainda, ao emprego rotineiro generalizado da ultrassonografia durante a gravidez ${ }^{10}$. Entre os casos sintomáticos a queixa mais comum persiste sendo o sangramento ${ }^{12}$.

Devemos destacar, no entanto, que apesar do diagnóstico precoce, a frequência de doença persistente mesmo entre estes casos permanece a mesma ${ }^{11}$.

\section{Ultrassonografia e dosagens de gonadotrofinas coriônicas}

Apenas metade das gestações molares de primeiro trimestre têm a aparência clássica à ultrassonografia, que é a de múltiplos cistos de dimensões diferentes e ausência de embrião (ou embrião não-visível) ${ }^{13}$.

Tanto o desenvolvimento máximo das vesículas na cavidade uterina como a eventual eliminação das mesmas ocorrem durante o segundo trimestre. Neste período, a ultrassonografia pode mostrar o habitualmente mencionado aspecto de múltiplas vesículas, que é devido ao acentuado edema das vilosidades ${ }^{13}$. No entanto, como a maior parte dos casos é submetido a exame pela ultrassonografia no primeiro trimestre, esta característica pode não ser observada ${ }^{14-16}$. Outro aspecto que deve ser levado em consideração quando se analisa a acurácia do diagnóstico ultrassonográfico de $\mathrm{MH}$ em situações clínicas é o número reduzido de casos com esta complicação, em relação aos outros diagnósticos mais frequentemente associados ao sangramento no primeiro trimestre.

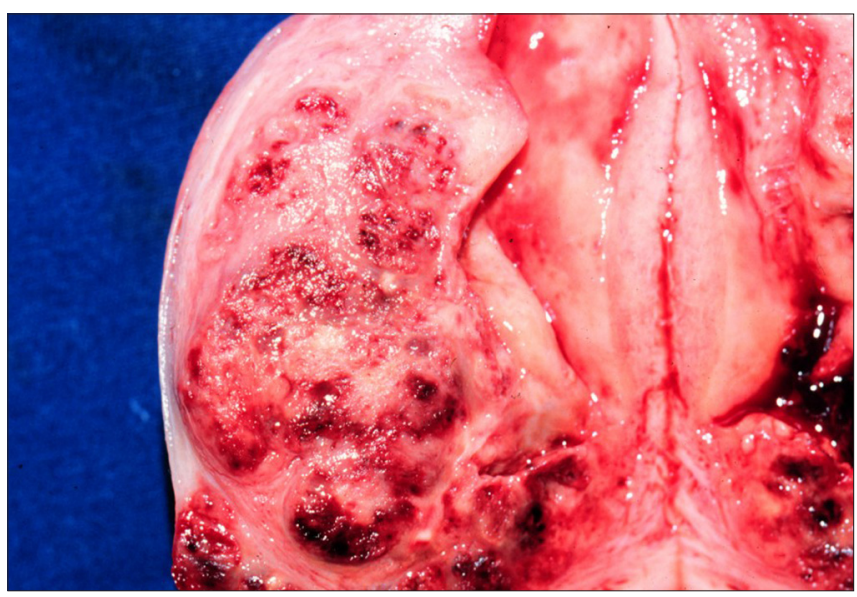

Figura 1 - Útero obtido por histerectomia abdominal indicada por hemoperitôneo. Invasão extensa do miométrio por mola invasora chegando até a serosa. Observar a cavidade uterina sem doença trofoblástica, o que explica sucessivas curetagens negativas para trofoblasto.
Em um grande centro de referência da região Sudeste do Brasil, a porcentagem de molas hidatiformes entre 115 casos de gestações inviáveis foi de apenas $0,8 \%{ }^{14}$. Por estes motivos a taxa de acertos da ultrassonografia é baixa, permitindo o diagnóstico em apenas 40 a $60 \%$ dos $\operatorname{casos}^{15}$. Os demais casos ficam com os diagnósticos de gestação anembrionada, abortamento incompleto ou abortamento retido ${ }^{13}$ até a resolução e avaliação histopatológica. A ultrassonografia pode contribuir para o diagnóstico de MHP. Alguns achados são considerados bastante sugestivos, como alterações císticas focais na placenta e aumento da relação entre o diâmetro transverso e o ântero-posterior. As modificações no formato do saco gestacional são indicativas da embriopatia relacionada à triploidia. Quando ambas as alterações estão presentes, o valor preditivo positivo chega a $90 \%{ }^{17}$. Outros achados relacionados à MHP são: a restrição do crescimento fetal e múltiplas malformações associadas à placenta focalmente hidrópica. A dosagem de gonodotrofinas coriônicas (hCG) pode complementar as informações da ultrassonografia, particularmente se os títulos forem superiores ao valor esperado para a idade gestacional. Os níveis de hCG entre as portadoras de MHC são bastante elevados e quase metade das pacientes têm níveis superiores a $100.000 \mathrm{mUI} / \mathrm{mL}^{18}$, podendo ser observados valores superiores a $5.10^{6} \mathrm{mUI} / \mathrm{mL}$. No entanto, os níveis são bem menores entre os casos com MHP e valores superiores a $100.000 \mathrm{mUI} / \mathrm{mL}$ são observados em apenas $10 \%$ dos casos $^{19}$.

\section{Diagnóstico de doença trofoblástica em pacientes sem diagnóstico prévio de mola}

Eventualmente, pacientes com $\mathrm{MH}$ ou suas sequelas apresentam quadros clínicos atípicos, diferentes dos descritos neste artigo. Geralmente estas situações ocorrem após abortamento molar no qual o diagnóstico histológico não é obtido. A forma mais frequente de apresentação é o sangramento de repetição e, sucessivas curetagens não mostram a presença de material trofoblástico (Figura 1). Outras vezes, em decorrência da perfuração do miométrio por mola invasora, o quadro é de hemoperitôneo. No entanto, é preciso estar atento para outras formas, como hemoptise, nódulos pulmonares, lesões expansivas no sistema nervoso central, entre outras. Em todas estas situações, em mulheres em idade fértil deve ser solicitada a dosagem sérica ou urinária da gonadotrofina coriônica ${ }^{20}$.

Para se evitarem estas situações, frequentemente associadas à neoplasia trofoblástica de alto risco, todo material obtido por curetagem ou aspiração de produto da concepção de gestações não-viáveis deve ser submetido à análise histopatológica. Cerca de $70 \%$ das molas parciais e $15 \%$ das MHCs somente são diagnosticadas 
pela avaliação histopatológica do material obtido por curetagem ou vácuo-aspiração ${ }^{21}$. Se o diagnóstico não é feito nesta fase, a paciente não é seguida com dosagens de gonadotrofinas e pode ter complicações potencialmente ameaçadoras da vida, como descrito acima, e com mais frequência necessitarão de cirurgia e quimioterapia ${ }^{20}$.

\section{Abordagem inicial dos casos com} suspeita ou diagnóstico ultrassonográfico de mola hidatiforme

Quando há suspeita ultrassonográfica ou clínica de MHC ou MHP, o conteúdo da cavidade uterina deve ser esvaziado segundo técnica resumida adiante. Deve ser evitado o uso de drogas que provoquem contrações uterinas, pois sabemos que a indução de contrações uterinas antes do esvaziamento da mola aumenta o risco de evolução para doença persistente $e^{22,23}$ e de embolização trofoblástica para vasos pulmonares ${ }^{23}$. Para o esvaziamento, é recomendada a vácuo-aspiração, devendo ser evitada a curetagem no início do procedimento, pois o risco de perfuração é elevado ${ }^{24}$. Além disso, no caso de molas de grande volume, o tempo para se efetuar uma curetagem pode ser muito longo, o que aumenta a perda sanguínea. $\mathrm{O}$ procedimento deve ser completado com a curetagem das paredes uterinas para confirmar a remoção completa do material molar. O material obtido pela curetagem apresenta menor índice de autólise e necrose, sendo mais adequado para histopatologia, devendo ser enviado separadamente.

Até há pouco tempo não se recomendava nova aspiração ou curetagem após o procedimento inicial. No entanto, dois estudos com grande casuística mostraram que em certas circunstâncias esta segunda intervenção pode ser benéfica ${ }^{25,26}$, levando à negativação dos títulos de gonadotrofinas ou controlando sangramento. Em um destes estudos, 282 pacientes classificadas como portadoras de doença persistente (subunidade $\beta$ de hCG detectável após quatro a seis semanas de evolução) foram submetidas à segunda curetagem, das quais $171(60 \%)$ foram curadas após o procedimento ${ }^{25}$.

No entanto, há riscos associados a esta segunda intervenção, dos quais o mais importante é a perfuração uterina. Para a indicação do procedimento, alguns critérios devem ser verificados: paciente com sintomas (sangramento) e manutenção dos níveis de gonadotrofina coriônica; volume uterino aumentado ou achado ultrassonográfico de material molar intracavitário, mas sem imagens sugestivas de mola invasora. A segunda curetagem deve ser contraindicada se houver metástases, elevação persistente dos níveis de hCG e diagnóstico de coriocarcinoma, situações em que o tratamento pela quimioterapia é prioritário.

Uma alternativa para a abordagem inicial é a histerectomia, pouco empregada atualmente. Pode ser oferecida a pacientes com mais de 40 anos, entre as quais o risco para doença invasora é maior. A histerectomia elimina o risco da doença invasora, mas não o de doença metastática.

Antes do esvaziamento da cavidade uterina, a paciente deve ser submetida à avaliação clínica com destaque para o diagnóstico de eventuais complicações, como hipertireoidismo, indícios de insuficiência respiratória, anemia e pré-eclampsia. Todas estas situações devem ser corrigidas antes do procedimento. No caso de hipertireoidismo, empregar betabloqueadores antes da indução da anestesia para se evitar a crise tireotóxica. É recomendada para todos os casos a radiografia de tórax e coleta de sangue para dosagem quantitativa da subunidade $\beta$ hCG. Pacientes $\mathrm{Rh}$ negativas devem receber imunoglobulina nas doses habituais ao tempo do esvaziamento, visto que o trofoblasto expressa o fator RhD.

\section{Seguimento após o tratamento} da mola hidatiforme

O objetivo mais importante do seguimento é detectar precocemente os casos que apresentem persistência da doença. Desta forma, a maioria deles será classificado como de baixo risco, podendo ser tratados com monoquimioterapia, e apresentando prognóstico melhor ${ }^{27}$. As recomendações atuais para o seguimento preveem a dosagem semanal de hCG até que haja três dosagens consecutivas negativas. $\mathrm{O}$ valor abaixo do qual o teste é considerado negativo varia, mas em geral é inferior a $5 \mathrm{mUI} / \mathrm{mL}$. Em seguida, as dosagens podem ser mensais por 6 a 12 meses $^{28}$, sendo o seguimento por um ano reservado para as pacientes tratadas com monoquimioterapia ${ }^{29}$ para doença persistente de baixo risco.

Os valores obtidos devem ser comparados com os da semana anterior e, para os casos com evolução espontânea, há regressão constante dos níveis. A porcentagem de redução entre as dosagens sucessivas deve ser estabelecida pelos serviços. É discutido também o emprego de curvas padrão para avaliação da regressão dos níveis de $\beta$ hCG. Já foi demonstrado que pacientes que apresentam dosagens que superam a linha padrão têm risco mais elevado de evolução para formas malignas e, que este achado pode antecipar o diagnóstico ${ }^{30,31}$. No entanto, este achado não é suficiente para a indicação de tratamento sistêmico e estudos com maior casuística são necessários para comprovar o papel da curva de regressão normal da $\beta h C^{31}$.

A dosagem de $\beta$ hCG não é, porém, o único componente do seguimento. As pacientes devem ser avaliadas clinicamente no início do seguimento, mas não é recomendada a repetição dos exames ginecológicos nos retornos. Deve ser solicitada radiografia de tórax (a não ser que um exame negativo tenha sido obtido antes do esvaziamento). Outros exames de imagem, como ultrassonografia, não 
são necessários, a não ser que haja sangramento anormal ou para o estadiamento no caso com elevação dos níveis de gonadotrofinas. Queixas como sangramento devem ser valorizadas e a causa, investigada (ver figura 2). É importante a adesão da paciente a um método contraceptivo eficiente, sendo preferidos os contraceptivos orais e os injetáveis. A ideia difundida nos anos 1970 de que os contraceptivos aumentariam o risco de doença persistente não foi confirmada posteriormente ${ }^{32,33}$.

Para os casos com evolução espontânea, o seguimento por seis meses após a negativação das dosagens de $\beta$ hCG é considerado adequado. Após a introdução das técnicas sensíveis para dosagem e o uso de avaliações quantitativas foi observado que o risco de recorrência após negativação dos níveis de $\beta$ hCG é muito baixo para casos com MHC e $\mathrm{MHP}^{34,35}$. Um estudo que envolveu 1.029 mulheres com MHC demonstrou que não houve doença persistente ou metastática entre as que apresentaram regressão espontânea dos níveis para valores inferiores a $5 \mathrm{mUI} / \mathrm{mL}^{36}$. Outro estudo, com 238 pacientes portadoras de MHP, mostrou, também, que não houve casos com neoplasia trofoblástica após negativação da hCG plasmático ${ }^{35}$.

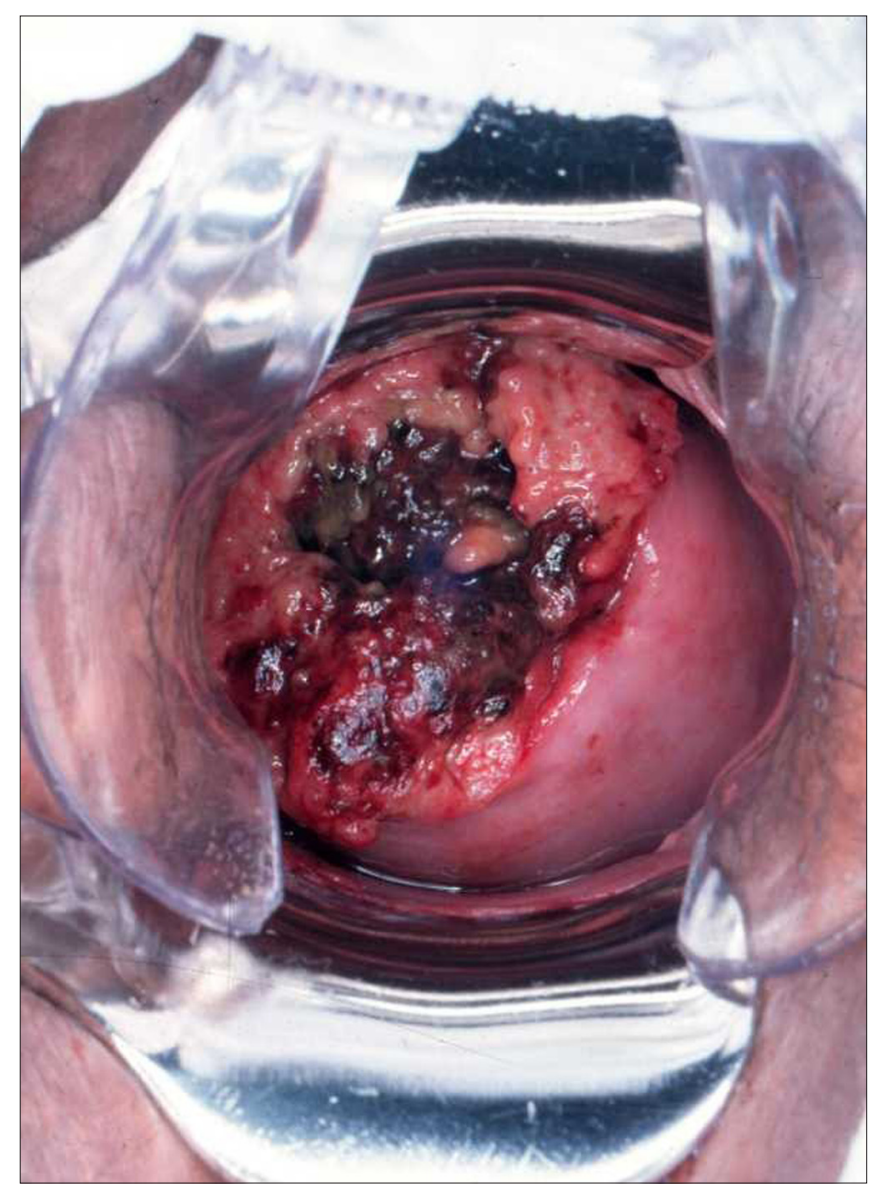

Figura 2 - Exame especular mostrando lesão cervical extensa, simulando carcinoma de colo. Estas lesões sangram intensamente após biópsia e, por este motivo, havendo suspeita de que se trata de doenças trofoblásticas gestacionais, efetuar a biópsia em ambiente de centro cirúrgico.
Por outro lado, a experiência de todos os serviços especializados mostra dificuldade para manter a aderência das pacientes a um seguimento ${ }^{29,37-38}$, especialmente para os mais longos. A redução no tempo de seguimento, além da maior adesão das pacientes e redução da ansiedade, levaria a uma importante diminuição nos custos. A introdução desta rotina implicaria reduzir o tempo de seguimento para aproximadamente $85 \%$ dos casos que apresentam evolução espontânea após a resolução da mola hidatiforme. Protocolos mais curtos, com alta logo após a negativação, têm sido propostos, principalmente para pacientes com MHP, para as quais o risco de evolução para invasão do miométrio e metástases é menor $^{39}$, mas a opinião dominante atualmente é que até que se confirmem estes resultados, deve-se continuar com o seguimento por pelo menos seis meses.

\section{Métodos para dosagem da gonadotrofina coriônica (subunidade $\beta$ )}

Para cumprir o papel de marcador tumoral no seguimento de pacientes com MH, a hCG deve ser dosada com técnica de laboratório capaz de detectar todas as suas formas ${ }^{40,41}$. O método deve apresentar sensibilidade e especificidade próximas a $100 \%{ }^{42}$. A decisão sobre qual material (soro ou urina) deve ser testado é também fundamental. No soro de gestantes normais, as concentrações da fração $\beta$ hCG são 200 vezes menores que a concentração da molécula de hCG completa, mas na urina a fração $\beta$ hCG é uma das formas predominantes desde o início da gravidez ${ }^{43}$. A subunidade $\beta$ é predominante em certas formas da doença trofoblástica, principalmente no caso de coriocarcinoma, mas tanto com o objetivo de diagnóstico como no seguimento de situações anormais, todas as outras formas devem ser quantificadas. Há uma clara correlação entre os níveis plasmáticos e urinários da molécula intacta de hCG e da subunidade alfa, entretanto, não há a mesma relação quanto à subunidade beta. Isto significa que haverá resultados discrepantes de acordo com o fluido analisado ${ }^{43}$. Portanto, para resultados coerentes, a molécula de hCG intacta deve ser quantificada em amostras de soro ou plasma, e não da urina ${ }^{40}$.

A hCG é uma glicoproteína composta de duas subunidades (alfa e beta) mantidas unidas por ligações nãocovalentes. A subunidade alfa é a mesma encontrada no hormônio luteinizante ( $\mathrm{LH}$ ) e outros, mas a subunidade beta é diferente em cada um deles. Há três formas mais importantes, quantitativamente, da hCG: a forma regular, a hiperglicosilada e a subunidade beta, derivada da segunda ${ }^{44}$. Entre as aplicações das dosagens de hCG está o monitoramento de vários tumores ${ }^{45}$. A forma hiperglicosilada é produzida pelo citotrofoblasto indiferenciado, cuja função fisiológica é manter o potencial invasivo na 
fase de implantação. A mesma função é desempenhada no caso da mola invasora ${ }^{45}$. A subunidade beta é produzida por vários tumores em quantidades variáveis ${ }^{45}$. Para o monitoramento da evolução dos casos de $\mathrm{MH}$ e avaliação da resposta ao tratamento quimioterápico, no entanto, todas as formas devem ser incluídas na dosagem. Esta informação deve ser lembrada porque a maioria dos testes comerciais para quantificar as hCG são limitados a molécula regular (intacta). Esta abordagem é devida parcialmente à postura do Food and Drug Administration, que considera a dosagem de hCG apenas como um teste para gravide $z^{45}$. Os testes disponíveis em todo o mundo, os mesmos empregados no Brasil, são bastante heterogêneos quanto às formas das moléculas quantificadas ${ }^{46}$, o que explica as divergências entre dosagens efetuadas em laboratórios diferentes. É claro, portanto, que os testes que avaliam a presença de apenas uma forma de hCG não são suficientes para as finalidades aqui discutidas. Assim, é preciso verificar com os laboratórios se o teste é adequado para o seguimento de pacientes com $\mathrm{MH}$ ou das tratadas com quimioterapia.

\section{Risco para doença persistente}

A doença persistente é caracterizada ou definida pela manutenção ou elevação dos níveis de hCG. Embora alguns empreguem este termo apenas quando não há metástases, este pode ser usado nas duas situações: doença metastática ou mola invasora. Esta última, por sua vez, se caracteriza por invasão do miométrio e dos seus vasos por vilosidades e somente pode ser confirmada em espécimes de histerectomia ${ }^{47}$. Clinicamente é, portanto, um diagnóstico de exclusão. Nesta revisão empregamos estes termos, além da expressão "neoplasia trofoblástica", para todas as sequelas que exigem tratamento quimioterápico.

O risco de evolução para doença persistente varia na dependência de vários fatores, incluindo o critério empregado para definição. De modo geral, a porcentagem de casos que necessitam de tratamento sistêmico para resolução e negativação das dosagens de gonadotrofina coriônica é de 15 a $20 \%$ dos casos com MHC e de 0,5 a $1 \%$ no caso de $\mathrm{MHP}^{48}$. Com base em várias características, é possível definir um grupo com risco mais elevado para evolução para doença persistente ${ }^{49}$. O risco para evolução para doença persistente (invasora ou metastática) é aumentado entre as pacientes que apresentam sinais indicativos de hiperplasia do trofoblasto, representados principalmente pela altura uterina maior que a esperada para a idade gestacional, presença de cistos tecaluteínicos ${ }^{50}$ e a dosagem sérica elevada de $\beta \mathrm{hCG}(\mathrm{OR}=4,8)^{49}$. Estas informações, no entanto, não devem ser empregadas para selecionar casos que devem ser seguidos com dosagens de hCG, pois se recomenda que todas as portadoras de MHP e MHC sejam submetidas ao seguimento padrão.

\section{Quimioterapia profilática}

O emprego da quimioterapia profilática tem sido discutido desde que foi proposto há algumas décadas. Estudos prospectivos randomizados evidenciaram claramente que esta intervenção reduz acentuadamente a frequência de doença invasora e metastática ${ }^{51}$. Neste estudo, entre as mulheres com $\mathrm{MHC}$ e consideradas de alto risco, a porcentagem de casos com doença persistente e/ou metastática foi reduzida de 47 para 14\%. No entanto, não houve alteração nestas porcentagens entre os casos considerados de baixo risco ${ }^{51}$. Foram obtidos resultados similares em outro estudo, nos quais se empregou actinomicina $\mathrm{D}$, ou seja, redução significativa da taxa de doença persistente apenas para o grupo com MHC considerado de alto risco: de $50 \%$ para $13,8 \%{ }^{52}$. Em um estudo não-randomizado e controlado, no qual foram incluídas apenas adolescentes que apresentavam alto risco de evolução para malignidade, foi empregado um pulso de 1,25 $\mathrm{mg}$ de actinomicina $\mathrm{D}$. No Grupo Tratado, a frequência de neoplasia trofoblástica foi de 6,9 contra 29\% no Grupo Controle ${ }^{53}$.

No entanto, a estes resultados positivos deve-se contrapor o custo da medicação, os efeitos colaterais imediatos e tardios e a consideração de que, entre 60 e $80 \%$ dos casos não teriam indicação para quimioterapia. Somente nas situações em que o seguimento não fosse viável estaria indicada a intervenção ${ }^{54}$.

\section{Referências}

1. Steigrad SJ. Epidemiology of gestational trophoblastic diseases. Best Pract Res Clin Obstet Gynaecol. 2003;17(6):837-47.

2. Sun SY, Amed AM, Bertini AM, Camano L. Incidência da mola hidatiforme na Escola Paulista de Medicina. Rev Assoc Med Bras. 1992;38(4):217-20.

3. Garner El, Goldstein DP, Feltmate CM, Berkowitz RS. Gestational trophoblastic disease. Clin Obstet Gynecol. 2007;50(1):1 12-22.
4. Howat A, Beck S, Fox H, Harris SC, Hill AS, Nicholson CM, et al. Can histopathologists reliably diagnose molar pregnancy? J Clin Pathol. 1993;46(7):599-602.

5. Paradinas FJ, Browne P, Fisher RA, Foskett $M$, Bagshawe KD, Newlands E. A clinical, histopathological and flow cytometric study of 149 complete moles, 146 partial moles and 107 non-molar hydropic abortions. Histopathology. 1996;28(2):101-10. 
6. Messerli ML, Parmley T, Woodruff JD, Lilienfeld AM, Bevilacqua $L$, Rosenshein NB. Inter- and intra-pathologist variability in the diagnosis of gestational trophoblastic neoplasia. Obstet Gynecol. 1987;69(4):622-6.

7. Zaragoza MV, Surti U, Redline RW, Millie E, Chakravarti A, Hassold TJ. Parental origin and phenotype of triploidy in spontaneous abortions: predominance of diandry and association with partial hydatidiform mole. Am J Human Genet. 2000;66(6):1807-20.

8. Matsui H, litsuka Y, Suzuka K, Seki k, Sekiya S. Subsequent pregnancy outcome in patients with spontaneous resolution of HCG after evacuation of hydatidiform mole: comparison between complete and partial mole. Hum Reprod. 2001;16(6):1274-7.

9. Soto-Wright V, Bernstein M, Goldstein DP, Berkowitz RS. The changing clinical presentation of complete molar pregnancy. Obstet Gynecol. 1995;86(5):775-9.

10. Belfort $P$, Braga A. Mudanças na apresentação clínica da gravidez molar. Rev Bras Ginecol Obstet. 2004;26(6):483-8.

11. Mangili G, Garavaglia E, Cavoretto P, Gentile C, Scarfone G, Rabaiotti E. Clinical presentation of hydatidiform mole in northern Italy: has it changed in the last 20 years? Am J Obstet Gynecol. 2008;198(3):302.e1-4.

12. Gemer O, Segal S, Kopmar A, Sassoon E. The current clinical presentation of complete molar pregnancy. Arch Gynecol Obstet. 2000;264(1):33-4.

13. Benson CB, Genest DR, Bernstein MR, Soto-Wright V, Goldstein DP, Berkowitz RS. Sonographic appearance of first trimester complete hydatidiform moles. Ultrasound Obstet Gynecol. 2000; 16(2): 188-91.

14. Watanabe LC, Brizot ML, Pereira PP, Mustafá SA, Miyadahira S, Zugaib M. Achados ultra-sonográficos em pacientes com ameaça de abortamento no primeiro trimestre da gestação. Rev Bras Ginecol Obstet. 2000;22(5):275-9.

15. Johns J, Greenwold N, Buckley S, Jauniaux E. A prospective study of ultrasound screening for molar pregnancies in missed miscarriages. Ultrasound Obstet Gynecol. 2005;25(5):493-7.

16. Fowler DJ, Lindsay I, Seckl M, Sebire NJ. Routine pre-evacuation ultrasound diagnosis of hydatidiform mole: experience of more than 1000 cases from a regional referral center. Ultrasound Obstet Gynecol. 2006;27(1):56-60.

17. Fine C, Bundy AL, Berkowitz RS, Boswell SB, Berezin AF, Doubilet PM. Sonographic diagnosis of partial hydatidiform mole. Obstet Gynecol. 1989;73(3 Pt 1):414-8.

18. Genest DR, Laborde O, Berkowitz RS, Goldstein DP, Bernstein MR, Lage J. A clinicopathologic study of 153 cases of complete hydatidiform mole (1980-1990): histologic grade lacks prognostic significance. Obstet Gynecol. 1991;78(3 Pt 1):402-9.

19. Berkowitz RS, Goldstein DP, Bernstein MR. Natural history of partial molar pregnancy. Obstet Gynecol. 1985;66(5):677-81.

20. Seckl M, Gillmore R, Foskett M, Sebire NJ, Rees H, Newlands ES. Routine terminations of pregnancy - should we screen for gestational trophoblastic neoplasia? Lancet. 2004;364(9435):705-7.

21. Lindholm H, Flam F. The diagnosis of molar pregnancy by sonography and gross morphology. Acta Obstet Gynecol Scand. 1999;78(1):6-9.

22. Andrade JM. Avaliação de diferentes técnicas de esvaziamento uterino como fator de risco para neoplasia trofoblástica invasora e metástica. Rev Assoc Med Bras. 1989;35(5):175-8.

23. Tidy JA, Gillespie AM, Bright N, Radstone CR, Coleman RE, Hancock BW. Gestational trophoblastic disease: a study of mode of evacuation and subsequent need for treatment with chemotherapy. Gynecol Oncol. 2000;78(3 Pt 1):309-12.
24. Tidy JA, Hancock BW, Newlands ES. The management of gestational trophoblastic neoplasia. Clinical guideline 38. [Internet]. London: Royal College of Obstetricians and Gynaecologists; 2004 [cited 2008 Nov 12]. Available from: http://www.rcog.org.uk/files/rcog-corp/ uploaded-files/GT38GestationalTrophNeoplasia 2004.pdf

25. Pezeshki M, Hancock BW, Silcocks P, Everard JE, Coleman J, Gillespie $A M$, et al. The role of repeat uterine evacuation in the management of persistent gestational trophoblastic disease. Gynecol Oncol. 2004;95(3):423-9.

26. Savage $P$, Seckl M. The role of repeat uterine evacuation in trophoblast disease. Gynecol Oncol. 2005;99(1):251-2.

27. McNeish IA, Strickland S, Holden L, Rustin GJ, Foskett M, SeckI M, et al. Low-risk persistent gestational trophoblastic disease: outcome after initial treatment with low-dose methotrexate and folinic acid from 1992 to 2000. J Clin Oncol. 2002;20(7):1838-44.

28. Hurteau JA. Gestational trophoblastic disease: management of hydatidiform mole. Clin Obstet Gynecol. 2003;46(3):557-69.

29. Allen JE, King MR, Farrar DF, Miller DS, Schorge JO. Postmolar surveillance at a trophoblastic disease center that serves indigent women. Am J Obstet Gynecol. 2003;188(5):1151-3.

30. Behtash N, Ghaemmaghami F, Honar H, Riazi K, Nori A, Modares $M$, et al. Is normal beta-hCG regression curve helpful in the diagnosis of persistent trophoblastic disease? Int J Gynecol Cancer. 2004; 14(5):980-3

31. Delmanto LRMG, Maestá I, Braga Neto AR, Michelin OC, Passos JRS, Gaiotto FR, et al. A curva de regressão da gonadotrofina coriônica humana é útil no diagnóstico precoce da neoplasia trofoblástica gestacional pós-molar? Rev Bras Ginecol Obstet. 2007;29(10):506-10.

32. Curry SL, Schlaerth JB, Kohorn El, Boyce JB, Gore H, Twiggs LB, et al. Hormonal contraception and trophoblastic sequelae after hydatidiform mole (a Gynecologic Oncology Group Study). Am J Obstet Gynecol. 1989; 160(4):805-9.

33. Costa HL, Doyle P. Influence of oral contraceptives in the development of post-molar trophoblastic neoplasia: a systematic review. Gynecol Oncol. 2006; 100(3):579-85.

34. Feltmate CM, Batorfi J, Fulop V, Goldstein DP, Doszpod J, Berkowitz RS. Human chorionic gonadotropin follow-up in patients with molar pregnancy: a time for reevaluation. Obstet Gynecol. 2003; 101(4):732-6.

35. Wolfberg AV, Growdon WB, Feltmate CM, Goldstein DP, Genest $D R$, Chinchilla $M E$, et al. Low risk of relapse after achieving undetectable HCG levels in women with partial molar pregnancy. Obstet Gynecol. 2006; 108(2):393-6

36. Wolfberg AJ, Feltmate CM, Goldstein DP, Berkowitz RS, Lieberman E. Low risk of relapse after achieving undetectable HCG levels in women with complete molar pregnancy. Obstet Gynecol. 2004; 104(3):551-4.

37. Uberti EMH, Diestel MCF, Lubbe LP, Zubaran GM, Costa PL. Controle ambulatorial pós-molar: importância da motivação continuada e do atendimento personalizado na adesão da paciente ao tratamento. Rev Bras Ginecol Obstet. 1992;14(6):267-71.

38. Massad LS, Abu-Rustum NR, Lee SS, Renta V. Poor compliance with postmolar surveillance and treatment protocols by indigent women. Obstet Gynecol. 2000;96(6):940-4.

39. Wielsma S, Kerkmeijer L, Bekkers R, Pyman J, Tan J, Quinn M. Persistent trophoblast disease following partial molar pregnancy. Aust N Z J Obstet Gynaecol. 2006;46(2): 1 19-23.

40. Medeiros SF, Norman RJ. Formas moleculares da gonadotrofina coriônica humana: características, ensaios e uso clínico. Rev Bras Ginecol Obstet. 2006;28(4):251-63. 
41. Mitchell H, Seckl M. Discrepancies between commercially available immunoassays in the detection of tumour-derived hCG. Mol Cell Endocrinol. 2007;260-262:310-3.

42. Sebire NJ, Foskett M, Short D, Savage P, Stewart W, Thomson $M$, et al. Shortened duration of human chorionic gonadotrophin surveillance following complete or partial hydatidiform mole: evidence for revised protocol of a UK regional trophoblastic disease unit. BJOG. 2007;114(6):760-2.

43. Norman RJ, Menabawey M, Lowings C, Buck RH, Chard T. Relationship between blood and urine concentrations of intact human chorionic gonadotropin and its free subunits in early pregnancy. Obstet Gynecol. 1987;69(4):590-3.

44. Cole LA. New discoveries on the biology and detection of human chorionic gonadotropin. Reprod Biol Endocrinol. 2009;7:8.

45. Cole LA. Human chorionic gonadotropin and associated molecules. Expert Rev Mol Diagn. 2009;9(1):51-73.

46. Cole LA, Shahabi S, Butler SA, Mitchell H, Newlands ES, Behrman HR, et al. Utility of commonly used commercial human chorionic gonadotropin immunoassays in the diagnosis and management of trophoblastic diseases. Clin Chem. 2001;47(2): 308-15.

47. Cheung AN. Pathology of gestational trophoblastic diseases. Best Pract Res Clin Obstet Gynaecol. 2003;17(6):849-68.
48. Seckl M, Fisher RA, Salerno G, Rees H, Paradinas FJ, Foskett M, et al. Choriocarcinoma and partial hydatidiform moles. Lancet. 2000;356(9223):36-9.

49. Tiezzi DG, Andrade JM, Reis FJC, Lombardi W, Marana HRC. Fatores de risco para doença trofoblástica gestacional persistente. Rev Bras Ginecol Obstet. 2005;27(6):33 1-9.

50. Maestá I, Rudge MVC, Abreu ES, Dalben I, Peraçoli JC. Preditores clínicos e histopatológicos de tumor trofoblástico gestacional pós-mola hidatiforme completa. Rev Bras Ginecol Obstet. 2000;22(3):167-73.

51. Kim DS, Moon H, Kim KT, Moon YJ, Hwang YY. Effects of prophylactic chemotherapy for persistent trophoblastic disease in patients with complete hydatidiform mole. Obstet Gynecol. 1986;67(5):690-4.

52. Limpongsanurak S. Prophylactic actinomycin D for high-risk complete hydatidiform mole. J Reprod Med. $2001 ; 46(2): 110-6$.

53. Uberti EM, Diestel MC, Guimarães FE, De Nápoli G, Schmid H. Single-dose actinomycin $D$ : efficacy in the prophylaxis of postmolar gestational trophoblastic neoplasia in adolescents with high-risk hydatidiform mole. Gynecol Oncol. 2006;102(2):325-32.

54. Sivanesaratnam V. Management of gestational trophoblastic disease in developing countries. Best Pract Res Clin Obstet Gynaecol. $2003 ; 17(6): 925-42$ 